\section{Ablation of Underexpanded and Malapposed Coronary Stent: Optical Coherence Tomography in Detecting Struts Migration: A Case Report}

\section{Abstract}

Rotactional atherectomy represents an interesting and readily available opportunity to ablate under expanded and undilatable coronary stents forcefully implanted into ill prepared lesions (stent ablation). In this setting, the intracoronary imaging by OCT is an essential tool for a deep analysis in order of procedural planning, with comprehension of potential critical points.

We present a case of stent ablation of an under expanded stent with proximal struts malapposition. By OCT analysis with rendered imaging of the stent, we observed a singular OCT finding: the proximal migration in the left main of the struts of the malapposed part of the stent, proximal to the site of the under expansion and subsequent stent-ablation. No other similar finding was previously reported in literature. In this contest the OCT ausilium allowed without any doubt the correct identification of this potential dangerous feature and determined a change in the procedural strategy.

Keywords: Percutaneous coronary intervention; Coronary stent; Rotational atherectomy; Stent ablation; OCT; Bail-out strategies

Received: January 14, 2018; Accepted: March 09, 2018; Published: March 16, 2018

\section{Pignatelli Antonio*, Tiecco Fabio, Lafranceschina Carlo and Contegiacomo Gaetano}

Interventional Cardiology Unit, Mater Dei Hospital, Bari, Italy

Corresponding authors: Pignatelli Antonio

” apignatelli@hotmail.it

Interventional Cardiology Unit, Mater Dei Hospital, Bari, Italy.

Tel: $393395936679 /+390805076727$

Fax: +0805076713

Citation: Pignatelli A, Tiecco F, Lafranceschina C, Contegiacomo G (2018) Ablation of Under Expanded and Malapposed Coronary Stent: Optical Coherence Tomography in Detecting Struts Migration: A Case Report. Interv Cardiol J. Vol.4 No.1:2

\section{Introduction}

With the purpose to fix a suboptimal result after stent implantation, published case reports describe a notable nonconventional application of the rotactional atherectomy: using the burrs' sheer power to ablate under expanded and undilatable coronary stents forcefully implanted into ill-prepared lesions. This technique, known as "stent ablation", represents a bail-out method of successfully handling a troublesome complication such as the stent under expansion.

Wide evidence of increased rates of in-stent restenosis and thrombosis in case of stent under expansion or malapposition, also with new-generation drug-eluting stents $[1,2]$ are present in literature.

The two larger experiences of stent ablation actually reported in literature pointed out the feasibility of the procedure, although with conflicting post-procedural midterm mortality and major adverse cardiovascular events (MACE) $[3,4]$. So, every effort should be taken to avoid potential critical outcomes and keep procedure safe.

\section{Case Presentation}

We describe the case of a gentleman 50-years old, undergone to coronarography in our Cath lab for stable angina Canadian Cardiovascular Society (CCS) grade 3; two years before, he had been submitted to surgical myocardial revascularisation by left internal mammary artery (LIMA) for left anterior descending artery (LAD) and II diagonal branch, and a right mammary artery in Y-graft for I diagonal branch (D1) and circumflex artery (CX).

For the evidence of occlusion of the proximal tract of the $Y$-arterial graft, we decided to perform an angioplasty for a severe stenosis at the ostium of the I diagonal branch in the same procedure. By right radial approach, after selective engagement of the left main with a guide catether EBU 3.5 / $6 \mathrm{Fr}$, the stenosis was crossed with a BHW 0.014 inches wire, then dilated by a semi-compliant balloon of $2.0 \mathrm{~mm}$ diameter, with subsequent implantation 
of a zotarolimus-eluting stent $2.5 \times 14 \mathrm{~mm}$ expanded at 18 atmospheres (ATM). For an evident under expansion of the stent, a post-dilation with a non-compliant balloon of diameter 2.75 $\mathrm{mm}$, a further implantation of a sirolimus-eluting stent $3.0 \times 8 \mathrm{~mm}$ (partially embricated to the proximal edge of the previous), and a final post-dilation with a non-compliant balloon of diameter 3.0 $\mathrm{mm}$ expanded at very high pressure (28 ATM) were performed, without significant improvement in the expansion of the stents (Figure 1). The optical coherence tomography (OCT) analysis confirmed the angiographical evidence, with focal sub expansion (minimal stent area $2.43 \mathrm{~mm}^{2}$, residual area stenosis $63.7 \%$ ) and proximal stent malapposition (Figure 2).

So, we planned a second procedure the day-after, with the purpose to rectify this suboptimal result by the ablation of the stents in the under expanded segment by rotactional atherectomy. By femoral approach, after selective engagement of the left main with a EBU 3.5 $8 \mathrm{Fr}$ side-holes guide catheter, the stent-ablation was realised by rotational atherectomy with a $2.0 \mathrm{~mm}$ burr at 180000 RPM. By OCT with rendered imaging of the stent, the ablation of the under expanded part of the stents and the plaque modification were evident; a singular OCT finding, angiographically not so clear and evident, and immediate by the software rendering of the stent metallic struts, was the proximal migration in the left main of the struts of the malapposed part of the stent, proximal to the site of the under expansion and subsequent stent-ablation (Figure 3). No other similar findings are previously reported; the ablation of stents just implanted (with no time for endothelial or neo-intima proliferation), associated to the malapposition of the struts proximal to the undilatable lesion, were the determinant factor for this unexpected complication.

On the basis of these findings, after the angiographical evidence of the optimal expansion of a 3.0 non-compliant balloon, we provided to implant two zotarolimus-eluting stents of $3.0 \mathrm{~mm}$ and $4.0 \mathrm{~mm}$ diameter along left-main-proximal LAD-proximal D1, with an optimal angiographical result. Finally, the OCT analysis showed satisfactory expansion and apposition of the stents (Figure 4), through the distal part of the arterial graft, the D1 provided to the perfusion of the $C x$.

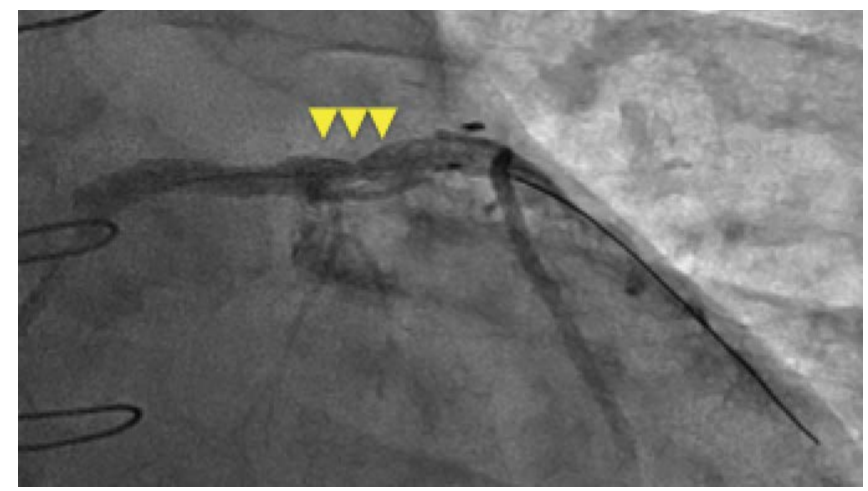

Figure 1 Angiographical evidence of underxpanded stent

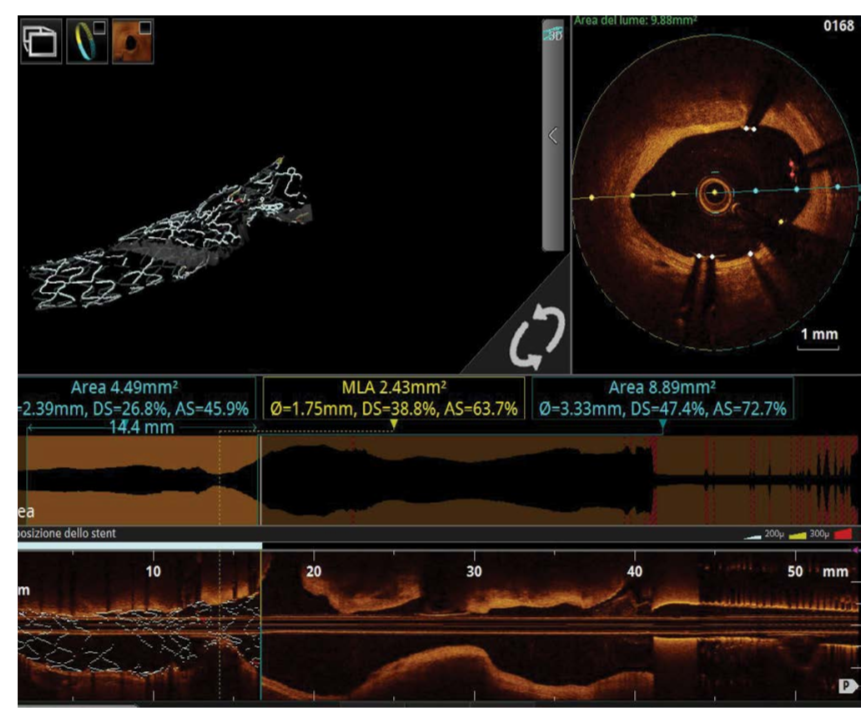

Figure 2 OCT analysis of underexpanded stent.

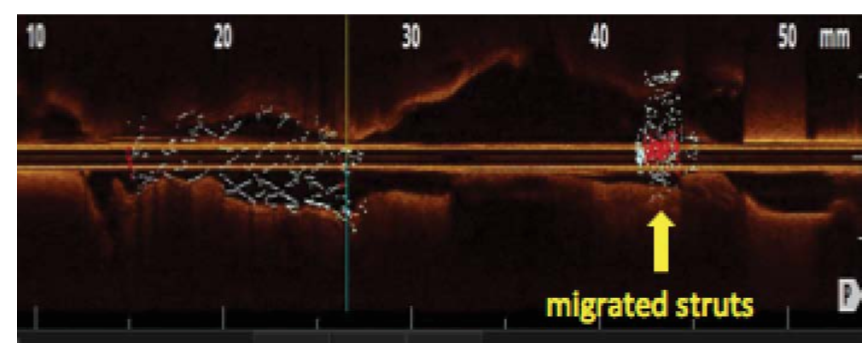

Figure 3 OCT findings after stentablation: longitudinal view with stent-rendering

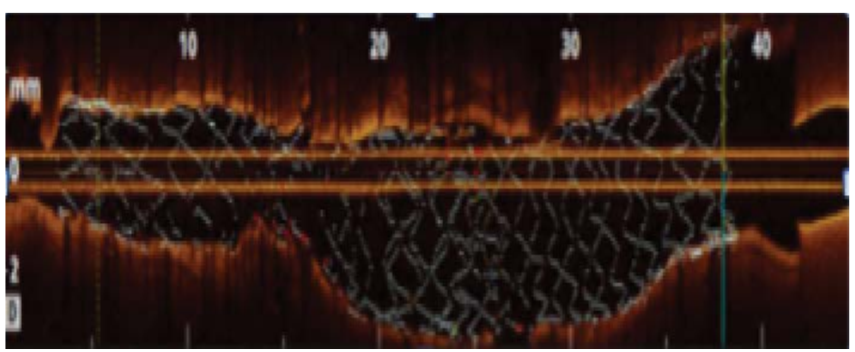

Figure 4 Final OCT findings.

\section{Discussion}

This case presented several challenging aspects. After a suboptimal result of the first percutaneous coronary intervention $(\mathrm{PCl})$, with a combination of sub expansion and malapposition, the stent-ablation allowed to correct this situation and, at the same time, to obtain the plaque modification necessary for the subsequent optimal stent implantation. In this specific setting, the malapposition proximal to the sub expansion determined an increased risk of burr entrapment during the stent ablation; for this reason, we provided to the distal positioning of the wire by a knuckle-shaped tip, in order to ensure a correct position of the wire in the lumen of the stents. 
It was remarkable the evidence of the proximal migration of ablated struts, as a consequence of the stent malapposition (in absence of neo-inthimal proliferation), and the necessary maneveuring of the burr; in this contest the OCT ausilium (powered by the software for the rendering of the metallic struts) allowed without any doubt the correct identification of this potential dangerous feature, and determined a change in the procedural strategy, with the implantation of a further stent along left-main-proximal LAD to crush and entrap the embolized struts.

In the evolution of percutaneous coronary interventions ( $\mathrm{PCI})$, the introduction of imaging systems contributes to provide clinically useful information on lesion significance, plaque risk burden and to optimize stent implant and prevent/manage periprocedural adverse events (restenosis, thrombosis, embolization) related to its position.

Optical coherence tomography (OCT) is a novel invasive imaging technique that produces high resolution intracoronary images. Its general principle of operation is similar to intravascular ultrasonography (IVUS); however, OCT uses infrared light, not ultrasounds. OCT (with an axial resolution of 10-20 micron whereas it is typically only $100-200$ micron with IVUS) is the gold

\section{References}

1 Taniwaki M, Radu MD, Zaugg S, Amabile N, Garcia-Garcia HM, et al. (2016) Mechanisms of very late drug-eluting stent thrombosis assessed by optical coherence tomography. Circulation 133: 650-660.

2 Windecker S, Meier B (2007) Late coronary stent thrombosis. Circulation 116: 1952-1965.

3 Édes IF, Ruzsa Z, Szabó G, Lux Á, Gellér L, et al. (2016) Rotational atherectomy of undilatable coronary stents: stentablation, a clinical perspective and recommendation. Eurolntervention 12: e632- e635.

4 Ferri LA, Jabbour RJ, Giannini F, Benincasa S, Ancona M, et al. (2016) standard to evaluate the results of coronary stenting in terms of expansion, apposition and edge dissections [5].

At the same time, OCT is a useful tool to guide the procedures, especially the more complex, and to identify possible dangerous points. In literature there is only one case of OCT-guided stentablation [6]; no similar finding of migration of part of the ablated stent (with OCT evidence) are reported.

\section{Conclusion}

In conclusion, as emerged by the review of the literature, the stent-ablation represents an interesting and readily available opportunity to fix a suboptimal result of the coronary stenting. In this setting, the intracoronary imaging by OCT is an essential tool for a deep analysis in order of procedural planning, with comprehension of potential critical points; obviously, the intracoronary imaging allows to document an optimal result (in terms of expansion and apposition of the struts, and individuation of residual edge dissection), fundamental after similar highrisk $\mathrm{PCl}$ with controversial mid-term outcomes. Randomized trials, although of paramount importance in the evidence-based medicine era, are difficult to achieve in this niche setting; the report of case series, present in literature, provide precious sorces of data and experiences.

Safety and efficacy of rotational atherectomy for the treatment of undilatable underexpanded stents implanted in calcific lesions. Catheter Cardiovasc Interv 90: E19-E24.

5 lannaccone M, D'Ascenzo F, Templin C, Omede P, Montefusco A, et al. (2016) Optical coherence tomography evaluation of intermediateterm healing of different stent types: A systemic review and metaanalysis. Eur Heart J Cardiovasc Imaging 18: 159-166.

6 Koide M, Inoue K, Matsuo A, Fujita H (2016) Optical coherence tomography findings after longitudinal ablation for an underexpanded stent in a heavily calcified lesion: A case report. BMC Cardiovascular Disorders 16: 241. 